\title{
Drug resistance tuberculosis: a global menace
}

Marahatta SB

Editor JMMIHS

Although progress has been made to reduce global incidence of drug-susceptible tuberculosis, the emergence of multidrug-resistant (MDR) and extensively drug-resistant (XDR) tuberculosis during the past decade threatens to undermine these advances [1]. The World Health Organization (WHO) has recognized M/XDR-TB as a major challenge to be addressed as part of the Stop TB strategy [2]. Patients infected with multiple-drug resistant strains are less likely to become cured particularly if they are infected by HIV or suffer from another immune disease. The treatment is much more toxic and much more expensive (about 700 times) than the one of patients with sensitive organisms [3]. A growing number of Mycobacterium tuberculosis (Mtb) strains are now resistant to the first-line anti- TB drugs, necessitating use of second-line drugs which are more expensive, less effective and more toxic [4]. Drug resistant TB originates from a range of human errors, including misuse of anti-TB drugs, poor adherence to treatment, poor-quality drugs and other reasons related to prescribers, patients and producers. Spontaneous chromosomal mutations at sites of key drug targets are the predominant mechanism of acquired resistance to antibiotic drugs by $M$. tuberculosis. Primary drug resistance - the transmission of already resistant $M$. tuberculosis - must be distinguished from secondary drug resistance - resistance acquired in the host as a result of inadequate treatment [5]. The global epidemic of drug-resistant tuberculosis is due to a combination of acquired resistance and primary transmission, although the relative contribution of each can only be estimated. Identification of the mechanisms through which drugresistant cases develop is important to establish the interventions necessary to prevent further cases.
Drug resistance can be definitely confirmed only with the aid of standardized, quality-controlled bacteriological sensitivity testing [6] phenotypically (i.e, culture growth in the presence of drug) or, more recently, genotypically (ie, identification of resistance-conferring mutations). However, at present neither technique is available in most countries because of a scarcity of capable laboratories [2].

Treatment of drug resistant tuberculosis requires the prolonged use of an antitubercular regimen comprised of at least four active drugs. Various treatment strategies have been employed, including the use of standardized treatment regimens based upon representative local susceptibility patterns, empirical treatment based upon previous treatment history and local DST patterns, and individualized treatment designed on the basis of individual DST results [7].

As there is a rise in trend of drug resistant TB with few treatment options available there is a concern over the treatment options for the future generation, hence it requires early detection of Drug resistant TB and its treatment with appropriate regimen based on drug resistant pattern from accredited laboratory [8]. Typically, such regimens include a fluoroquinolone and a secondline injectable agent (eg, amikacin, kanamycin, or capreomycin). Compared with first-line combination therapy, these regimens are less effective, require longer duration of therapy, and are often associated with serious toxicities and side effects - especially for coinfected patients receiving antiretroviral therapy (ART) [9]. Management of patients receiving second line 


\section{Editorial}

drugsrequires fairly intensive monitoring for drug toxicities and treatment failure. Sensitivity testing should be performed as rapidly as possible, particularly when drug resistance is suspected, so that the development of further resistance will not be promoted by nonspecific therapy. A single drug should never be added to an existing regimen, as this creates thedanger of monotherapy[6]. Treatment success rates are associated with degree of drug resistance; rates of success in MDR tuberculosis are as high as $83 \%$, and $60 \%$ in patients with XDR tuberculosis in the absence of HIV co-infection [2].

Drug resistant tuberculosis generally arises through the selection of mutated strains by inadequate therapy. The most successful approach to reduce the burden of drug resistant TB would be ensuring strong measures for tuberculosis case finding and compliance with DOTS amongst the identified cases. The ultimate strategy to control multidrug resistant tuberculosis is one that implements comprehensive approach incorporating treatment of multidrug-resistant tuberculosis based upon principles closely related to those of its general DOTS strategy for TB control: sustained political commitment; a rational case-finding strategy including accurate, timely diagnosis through quality assured culture and DST; appropriate treatment strategies that use second-line drugs under proper case management conditions; uninterrupted supply of quality-assured antitubercular drugs; standardized recording and reporting system. However, no technological or managerial innovation will make a meaningful difference to the response if access to care for the poorest and most vulnerable groups is not increased through strengthened and properly funded health-care systems.

\section{References}

1. Gandhi NR, Nunn P, Dheda K, Schaf S, Zignol M, Soolingen DV et al. Multi drug resistant and extensively resistant tuberculosis: a threat to global control of tuberculosis Lancet 2010;375:1830-43.

2. World Health Organisation. Towards universal access to diagnosis and treatment of multidrug-resistant and extensively drug-resistant tuberculosis by 2015: WHO progress report 2011.

3. Barroso EC, Mota RMS, Santos RO, Sousa AO, Barroso JB, Rodrigues JLN. Risk factors of acquired multidrug resistant tuberculosis J Pneumol 2003;29(2):89-97.

4. Fears R, Kaufmann S, Meulen V, Zumla A. Drug-resistant tuberculosis in the European Union: Opportunities and challenges for control. Tuberculosis 2010;90:182-7.

5. Miglior GB, Centis R, Lange C, Richardson MD, Sotgiu G. Emerging epidemic of drug-resistant tuberculosis in Europe, Russia, China, South America and Asia: current status and global perspectives. Current Opinion in Pulmonary Medicine 2010;16-20.

6. Loddenkemper R, Hauer B. Drug-Resistant. Deutsches Ärzteblatt International Dtsch Arztebl Int 2010; 107(1-2): 10-9.

7. World Health Organization. Guidelines for the Programmatic Management of Drug-Resistant Tuberculosis. Geneva: WHO, 2006.

8. Hafkin J, Gammino VM, Amon JJ. Drug-Resistant Tuberculosis in Sub-Saharan Africa. Curr Infect Dis Rep 2010; 12:36-45. 